\section{Utilidad de la crio-tecnología para uso diagnóstico y terapéutico en neumología intervencionista: crio-biopsia pulmonar transbronquial y crioterapia}

\author{
FRANCO MONTUFAR ${ }^{1}$, LUCRECIA DEL MORAL ${ }^{2, a}$, \\ GONZALO LABARCA ${ }^{3}$, ERIK FOLCH ${ }^{4}$, \\ ADNAN MAJID ${ }^{5}$, SEBASTIAN FERNANDEZ-BUSSY ${ }^{6}$
}

\section{Transbronchial cryobiopsies and cryotherapy in lung diseases}

Bronchoscopy cryoprobes are used for palliative treatment of endobronchial obstructions caused by tumors and removal of granulation tissue or foreign bodies. Currently this technology is also used for diagnosis of diffuse interstitial lung disease (ILD). The multidisciplinary team that establishes the clinical, radiological and histopathological correlation in ILD, decides about performing a surgical lung biopsy when the characteristics of the interstitial disease are not similar to Idiopathic Pulmonary Fibrosis (IPF). Although surgical lung biopsy is the gold standard for diagnosis, treatment, and prognosis, transbronchial cryo-biopsy has a high diagnostic yield, low morbidity and mortality rate, low rate of complications and lower cost. It is the diagnostic method of choice in ILD when it is available. Technological improvements with greater freezing power and tensile strength of the cryo probes, allow their use in cryotherapy and cryo-recanalization for occlusive airway tumors.

(Rev Med Chile 2018; 146: 1033-1040)

Key words: Biopsy; Diagnostic Techniques and Procedures; Idiopathic Pulmonary Fibrosis.
'Servicio de Neumología,

Universidad de Antioquia.

Colombia.

${ }^{2}$ Servicio de Neumología, Hospital

Universitario Fundacion Favarolo.

CABA. Argentina.

${ }^{3}$ Facultad de Medicina,

Universidad San Sebastián,

Concepción. Chile.

${ }^{4}$ Division of Pulmonary and Critical Care. Massachusetts

General Hospital-Harvard Medical

School, Boston. United States.

${ }^{5}$ Division of Interventional

Pulmonology and Thoracic

Surgery. Beth Israel Deaconess

Medical Center-Harvard Medical

School, Boston. United States.

${ }^{6}$ Division of Pulmonary and

Critical Care Medicine, Mayo

Clinic Jacksonville, Florida. United

States.

${ }^{a}$ Residente de Neumología.

Los autores declaran que el presente artículo no recibió financiamiento y no declaran

conflicto de interés.

Recibido el 4 de abril de 2018 , aceptado el 7 de agosto de 2018 .

Correspondencia a:

Dr. Gonzalo Labarca

Lientur 1457, Concepción,

Chile.

ORCID ID: 0000-0002-0069-

3420

glabarcat@gmail.com
E 1 uso de la crio-tecnología (CBPTB) en broncoscopia esta descrita desde $1977^{1-3}$, las criosondas fueron utilizadas inicialmente en la reducción de tumores que ocluían el lumen de la vía aérea carentes de efectos inmediatos, lo que conducía a necrosis de los tejidos requiriendo procedimientos broncoscópicos de limpieza posteriores. Las modificaciones en la técnicas en crio-tecnología generaron mayor poder de congelamiento y mayor fuerza de tensión condujeron al uso de esta tecnologia dentro del manejo paliativo de obstrucciones bronquiales por tumores de crecimiento exofítico y en la remoción de tejidos de granulación, cuerpos extraños y coágulos alojados en la vía aérea central ${ }^{3}$.

Sin embargo, el uso de la CBPTB como medio para la obtención de biopsias de parénquima pulmonar a través de broncoscopia, en especial la criobiopsia pulmonar transbronquial ha reportado ser de gran utilidad en el estudio de enfermedad pulmonar intersticial difusa (EPID), en especial cuando su clasificación diagnostica es dificultosa debido a los hallazgos tanto clínicos como tomo- 
gráficos no concluyentes, requiriendo muestras de tejido pulmonar para estudio histopatológico, obteniendo así un diagnóstico, pronóstico y tratamiento más preciso ${ }^{4-6}$.

El bajo rendimiento diagnóstico de las muestras de biopsia transbronquial convencional o con fórceps en EPID, que oscila entre $9 \%$ a $37 \%{ }^{7,8}$, hizo que la biopsia pulmonar quirúrgica (BPQ) sea recomendada como el estándar de oro. Sin embargo, la BPQ, ya sea por toracoscopía video asistida (VATS) o por toracotomía; tiene mayor morbilidad, riesgo de exacerbación de EPID $^{9,10}$, estancia hospitalaria promedio de 6,2 días y mortalidad a 30 días que oscila entre $2,7 \%$ a $21,7 \%{ }^{11,12}$.

Los estudios realizados a la fecha indican que la CBPTB es, actualmente, el procedimiento de elección para estudio de la EPID de causa no clara ${ }^{13,14}$.

Pretendemos con esta revisión, dar a conocer los aspectos técnicos de la CBPTB, otros usos diagnóstico y terapéutico actuales y resaltar los aspectos más importantes de los trabajos realizados.

\section{Aspectos técnicos}

El mecanismo de acción del equipo de crioterapia está basado en el efecto Joule-Thompson, donde la liberación de gas líquido comprimido a alto flujo en la punta de la criosonda se expande rápidamente y genera descenso térmico hasta $-75^{\circ} \mathrm{Ca}-89^{\circ} \mathrm{C}$. El gas utilizado (dióxido de carbono u óxido nitroso) es aplicado a altas presiones (45 bar) a través del canal central de la criosonda. El equipo Erbe Elektromedizin $\mathrm{GmBH}$, utiliza criosondas de $1.9 \mathrm{~mm}$ y $2.4 \mathrm{~mm}$ de diámetro, lo que define el tamaño de las muestras ${ }^{15-17}$.

El procedimiento es bajo sedación profunda o anestesia general, asegurando la vía aérea con tubo orotraqueal (TOT) N. ${ }^{\circ} \geq 8$, mascara laríngea N. ${ }^{\circ}$ $\geq 4$ o broncoscopio rígido, permitiendo extracción de la muestra y fácil reingreso.

Se evalúa la tolerancia a la oclusión bronquial para control de hemostasia que se realiza postbiopsia inmediata del bronquio lobar o segmentario del sitio elegido para la crio-biopsia ${ }^{18,19}$, ya sea con catéter Fogarty con balón, bloqueador endobronquial de Arndt ${ }^{\circledR}$ o balón para dilatación, que se puede introducir por externo al TOT, o por el canal lateral del mismo, o por el broncoscopio rígido externo al broncoscopio flexible.

Luego, se introduce la criosonda a través del broncoscopio flexible, bajo visión fluoroscópica, posicionándose aproximadamente a $2 \mathrm{~cm}$ de la superficie pleural (o cuando encuentra resistencia, se retira aproximadamente $1-1,5 \mathrm{~cm})^{20}$. Si queda en una ubicación más periférica existe un mayor riesgo de neumotórax (debido a la cercanía con la superficie pleural) y si se ubica más central puede aumentar el riesgo de sangrado (debido al tamaño de los vasos) $)^{17,18}$.

El tiempo de activación es de 5 a 7 segundos. La extracción se realiza en bloque, tanto del videobroncoscopio junto a la criosonda con el fragmento pulmonar congelado y adherido (puesto que el tamaño de la muestra no permite su retirada a través del canal de trabajo), el proceso se realiza mediante tracción firme y rápida para lograr desprendimiento de la muestra ${ }^{15,17,19}$. Finalmente, la punta de la sonda con la biopsia adherida es sumergida inmediatamente en un frasco con formalina a $10 \%$ y luego se lava el extremo distal de la sonda.

La continuidad de toma de muestras debe hacerse en ausencia de sangrado. El número de biopsias a tomar oscila habitualmente entre $4-6^{18}$.

Durante el procedimiento e inmediatamente finalizado, se realiza control con fluoroscopia, y en las primeras $3 \mathrm{~h}$ debe realizarse radiografía de tórax y ante síntomas o signos que sugieran la posibilidad de neumotórax ${ }^{18}$.

De manera complementaria, muchos centros están utilizando endosonografía endobronquial radial para guiar las biopsias en lesiones periféricas $^{21,22}$.

\section{Indicaciones}

Las siguientes son condiciones en las que la CBPTB ha demostrado ser de utilidad ${ }^{20,23}$ :

- Indicación de estudio para histopatología, en EPID evaluada como inconsistente.

- Biopsias de nódulos pulmonares periféricas de hasta $4 \mathrm{~cm}$ de diámetro.

- Manejo paliativo de obstrucción endobronquial por tumores de crecimiento exofítico; criobiopsia o crio-recanalización.

- Tratamiento de lesiones malignas de bajo grado y carcinoma in situ.

- Remoción de tejido de granulación en vía aérea

- Extracción de cuerpos extraños y remoción de coágulos. 


\section{Contraindicaciones}

Las siguientes son contraindicaciones para CBPTB $^{20,23}$ :

- Recuento de plaquetas $<50.000 / \mathrm{mm}^{3}$ o $>1$ millón $/ \mathrm{mm}^{3}$.

- Tiempo de Tromboplastina parcial activada $($ aPTT $)>50$ segundos.

- Ratio internacional normalizada (INR) $>1,5$.

- Anticoagulación no revertida o suspendida (warfarina 3 días, heparina de bajo peso molecular $12 \mathrm{~h}$, heparina fraccionada 4-6 h).

- Anti agregación no suspendida en los últimos 5-7 días, especialmente clopidogrel y los nuevos anticoagulantes (AAS no es necesaria suspenderla si la dosis no excede los $100 \mathrm{mg} /$ día).

- Uremia elevada, que afecta la función plaquetaria.

- Inestabilidad hemodinámica.

- Hipoxemia severa al aire ambiente; $\mathrm{PaO}_{2}$ $<55 \mathrm{mmHg}$.

- Crio-recanalización de la vía aérea por neoplasias de crecimiento extraluminal, con compresión extrínseca o tejidos crio-resistentes.

- Presión sistólica de arteria pulmonar $>40 \mathrm{~mm}$ Hg estimada por ecocardiografía, pero no está bien definido.

\section{Condiciones para realización de $\mathrm{CBPTB}^{20,23}$}

- Estudios de función pulmonar: Capacidad vital forzada mayor de $50 \%$ del valor normal predicho, VEF1 >0,8 L, Capacidad de difusión de monóxido de carbono (DLco) > 35\% del valor normal predicho.

- $\mathrm{PaO}_{2}>55 \mathrm{~mm} \mathrm{Hg}$ al aire ambiente,

- Conteo de plaquetas $>70.000$, INR $<1,5$.

\section{Características de las muestras en criobiopsia}

Cumplen con las características requeridas de una muestra para histopatología: tamaño adecuado, ausencia de artefactos, presencia de tejido alveolo-intersticial.

El rendimiento diagnóstico, según el diámetro de la criosonda, es 70\%-95\% con 2,4 mm $\mathrm{mm}^{9,17,24-26} \mathrm{y}$ $80 \%$ con $1,9 \mathrm{~mm}$ (promedio: $83,7 \%)^{16,26-29}$.

El tamaño de las muestras es suficiente para realizar los estudios histopatológicos habituales, además de conservar la estructura histológica preservada (carece de artefactos de aplastamiento $\mathrm{y}$ atelectasias generados por fórceps tradicional) permitiendo aplicar con éxito técnicas de inmunohistoquímica ${ }^{15}$.

\section{Complicaciones de la criobiopsia}

- Sangrado: En un meta-análisis publicado por Iftikart y cols $^{30}$ la incidencia de sangrado moderado a severo después de la CBPTB fue de $4,9 \%(2,2 \%$ a $10,7 \%)$. La severidad del sangrado, según descripciones realizadas en diferentes estudios podría catalogarse en 4

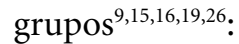

Grado 0: no sangrado.

Grado 1 o Leve: fácilmente controlado con succión/aspiración.

Grado 2 o Moderado: requiere uso de balón de bloqueo bronquial, suero fisiológico frío, adrenalina tópica, o procedimientos endoscópicos adicionales.

Grado 3 o Severo: amenaza la vida. Además del bloqueo bronquial y medidas descritas para el grupo II, requiere medidas adicionales como transfusiones, traslado a UCI. Embolización o intervención de cirugía de tórax.

- Neumotórax: incidencia de 9,5\% (5,9\% a $14,9 \%)$, en ocasiones requiere tubo de drenaje pleural para su resolución. Aunque se han reportado incidencia de hasta $30 \%^{16,17,26,30}$.

- Neumomediastino: especialmente en desbridamiento, con o sin presencia de neumotórax. Es raro, descrito en el 1,4\% de los casos ${ }^{19}$.

- Absceso pulmonar: complicación tardía en el sitio de biopsia, extremadamente raro y fue descrito en 1 paciente después de 2 semanas de la biopsia ${ }^{19}$.

- Muerte: se describe una mortalidad en 30 a 60 días con CBPTB de $0,7 \%$, mucho menor que la descrita para VATS $(1,8 \%)$, y en caso de EPID asociada a exacerbación ${ }^{11,17,26}$. Varía en caso de lesiones obstructivas de la vía aérea, con una mortalidad a 30 días de $7,1 \%$, cuyas principales causas fueron hemoptisis y falla respiratoria, relacionando la progresión de la enfermedad tratándose de neoplasia ${ }^{31}$. 


\section{Comparación con otras formas de toma de muestra de tejido pulmonar}

\section{- Criobiopsia vs biopsia transbronquial con fórceps (convencional)}

Estudios realizados previamente pusieron en evidencia el rol de la biopsia pulmonar transbronquial con fórceps; la sensibilidad y valores predictivos negativos fueron bajos; $30 \%$ y $50 \%$ respectivamente, relacionados con alta proporción de muestras pequeñas inadecuadas, no diagnósticas y con artefactos. La especificidad y valores predictivos positivos reportados en estos estudios fueron $96 \%$ y $95 \%{ }^{32-34}$.

Cuando se comparó la CBPTB con la biopsia transbronquial, los diagnósticos histopatológicos fueron mucho mayores en el grupo de criobiopsia; $74,4 \%$ vs $34,1 \%$ con una diferencia estadísticamente significativa $(\mathrm{p}<0,001)$, al igual que el rendimiento diagnostico $51,3 \%$ vs $29,1 \%$ $(\mathrm{p}=0,038)$. El área de las biopsias obtenidas por CBPTB fue mucho mayor $14,7 \pm 11 \mathrm{~mm}^{2}$ que las obtenidas con fórceps $3,3 \pm 4,1 \mathrm{~mm}^{2}$ con diferencia significativa $^{31-33}$.

El peso y diámetro de las muestras tomadas por CBPTB es $60 \%$ y $30 \%$ mayor que las obtenidas con fórceps. Presentando un diámetro entre $3,9 \mathrm{~mm}$ y $12 \mathrm{~mm}$ y un área promedio de $43,11 \mathrm{~mm}^{2}$ con mayor proporción de tejido alveolar $r^{9,16,26}$.

El sangrado grado 2 no tuvo diferencias significativas entre los dos procedimientos $56,4 \%$ vs $34,2 \%$. Tampoco hubo diferencias en otro tipo de complicaciones $^{9,15,16}$.

Un meta-análisis demostró diferencias estadísticamente significativas entre la CBPTB y la biospsia transbronquial con respecto al tamaño de las muestras, además el rendimiento diagnóstico fue mucho mayor $91,67 \%$ vs $73,13 \%$ con una razón de riesgo (RR) de 1,36; 95\% CI (1,16-1,59, $\mathrm{p}=0,0002)$. La incidencia de sangrado con la CВPTB fue de $23,7 \%$ vs $20,8 \%$ en el grupo de la biopsia tranbronquial, sin existir diferencias significativas $(p>0,05 ; p=0,068)$. Finalmente, la incidencia de neumotórax fue de $6 \%$ más alta que la del grupo de biopsia con fórceps ${ }^{8,15}$.

\section{- Criobiopsia vs VATS}

En un meta-análisis se comparo CBPTB vs VATS $^{30}$; el rendimiento diagnóstico fue $83,7 \%$ y 92,7, con una sensibilidad para CBPTB y VATS de $87 \%$ y $91 \%$ y una especificidad de $57 \%$ y
$58 \%$, respectivamente. El Odds ratio (OR) para el diagnóstico por CBPTB y VATS fue 25,53 y 21,06 y el área bajo la curva ROC fue 0,85 y 0,74 , respectivamente.

Respecto a las complicaciones, la incidencia de sangrado grado 2 posterior a CBPTB que requirió intervención broncoscópica fue 4,9\% y de neumotórax 9,5\%. El tiempo medio de hospitalización fue 2,6 días, mucho menor que de VATS que fue 6,1 días. La mortalidad a 30-60 días descrita para la CPTB es de $0,7 \%$ (0,4\% a $1,2 \%)$, mucho menor que la descripta para VATS que es de 1,8\% (1,0\% a $3 \%)^{11,30}$.

En estudios previos de BPQ, la mortalidad a 30 días fue del 3 a 4\%, hay riesgo de infección, exacerbación aguda de enfermedad pulmonar intersticial, presencia de fistulas broncopleurales hasta en $6,2 \%$, en este grupo, la estadia hospitalaria promedio fue de 6,2 días y hasta $57 \%$ de los pacientes tuvieron dolor costal en sitio quirúrgico 7 a 12 meses después del procedimiento ${ }^{35,36}$.

En un análisis de costo-efectividad, se aportan datos concernientes al ahorro de la CBPTB en $€ 953,09 /$ paciente que requieren procedimientos de BPQ ambulatoria y $€ 1925,29$ /paciente con hospitalización ${ }^{28}$.

\section{CBPTB en enfermedades pulmonares parenquimatosas difusas}

En el año 2013, se publicó la actualización del consenso de la ATS/ERS con la clasificación de las neumonías intersticiales idiopáticas (NII) (Tabla 1$)^{5}$.

También existe una amplia gama de enfermedades pulmonares parenquimatosas difusas no neoplásicas y no infecciosas no clasificadas como NII que se agrupan mejor bajo el término EPID. Este grupo más grande incluye enfermedades con hallazgos histopatológicos idénticos a aquellos en el grupo NII pero con etiología conocida, comúnmente enfermedad del tejido conectivo o exposiciones respiratorias ${ }^{17,19}$. Se consideran también parte de la clase más amplia de EPID a entidades como linfangioleiomiomatosis, sarcoidosis, histiocitosis de células de Langerhans y neumonía eosinofílica. Finalmente, también se encuentran con frecuencia procesos neoplásicos o infecciosos que se hacen pasar por supuestas EPID, caracterizados por anomalías difusas en las imágenes pulmonares. 
Tabla 1. Clasificación de enfermedades parenquimatosas difusas, modificado de consenso ATS/ERS 2013

\begin{tabular}{|c|c|c|c|}
\hline \multirow[t]{5}{*}{ Idiopáticas } & \multirow[t]{3}{*}{ Frecuentes } & Crónicas & $\begin{array}{l}\text { Fibrosis pulmonar idiopática (FPI) } \\
\text { Neumonía intersticial inespecífica (NINE) }\end{array}$ \\
\hline & & Asociadas a tabaco & $\begin{array}{l}\text { Bronquiolitis respiratoria asociada a enfermedad intersticial (BR/EPID) } \\
\text { Neumonía intersticial descamativa (NID) }\end{array}$ \\
\hline & & Agudas/subagudas & $\begin{array}{l}\text { Neumonía organizada criptogénica (NOC) } \\
\text { Neumonía intersticial aguda (NIA) }\end{array}$ \\
\hline & \multicolumn{2}{|l|}{ Raras } & $\begin{array}{l}\text { Neumonía intersticial linfoide idiopática (NILI) } \\
\text { Fibroelastosis pleuroparenquimatosa idiopática (FEPI) }\end{array}$ \\
\hline & \multicolumn{2}{|c|}{ No clasificable } & $\begin{array}{l}\text { Datos clínicos, radiológicos o patológicos inadecuados } \\
\text { Discordancia clínica, radiológica y/o patológica } \\
\text { - Tratamiento previo: resulta en alteración de hallazgos radiológicos o } \\
\text { histológicos } \\
\text { - Entidades nuevas o variantes inusuales de entidades reconocidas pero } \\
\text { no caracterizadas en el consenso ATS/ERS } \\
\text { Algunos patrones radiográficos o patológicos que pueden ser encontra- } \\
\text { dos en FPI }\end{array}$ \\
\hline \multicolumn{3}{|c|}{ De causa conocida o asociadas } & $\begin{array}{l}\text { Asociadas a enfermedades del colágeno } \\
\text { Asociadas a enfermedades hereditarias } \\
\text { Inducidas por fármacos o radioterapia } \\
\text { Por polvos inorgánicos }\end{array}$ \\
\hline \multicolumn{3}{|c|}{ Granulomatosas o con granulomas } & $\begin{array}{l}\text { Sarcoidosis } \\
\text { Alveolitis alérgica extrínseca o por hipersensibilidad } \\
\text { Granulomatosis con poliangeítis } \\
\text { Neumonía eosnofílica }\end{array}$ \\
\hline \multicolumn{3}{|c|}{ Otras formas } & $\begin{array}{l}\text { Linfangioleiomiomatosis (LAM) } \\
\text { Proteinosis alveolar } \\
\text { Microlitiasis alveolar } \\
\text { Histiocitosis de células de Langerhans } \\
\text { Amiloidosis } \\
\text { Otras }\end{array}$ \\
\hline
\end{tabular}

En la actualidad se recomienda un diagnóstico clínico consensuado por un comité multidisciplinario compuesto por neumólogos expertos, patólogos y radiólogos después de la revisión de datos clínicos, radiológicos y patológicos disponibles. La histología es el elemento clave cuando no se puede establecer un diagnóstico preciso, sobre todo en las EPID que presentan un patrón fibrótico, siendo la fibrosis pulmonar idiopática (FPI) la entidad más frecuente $(30 \%)$ y de peor pronóstico, caracterizada por un patrón histológico de neumonía intersticial usual (NIU). Se han definido los hallazgos radiológicos típicos que permiten el diagnóstico mediante TAC de tórax de alta resolución ${ }^{5,30}$. Otras entidades que pueden presentar patrón histológico de NIU son algunas enfermedades sistémicas, formas fibróticas de neumonía intersticial no usual, alveolitis alérgica extrínseca en fase crónica, neumonitis por fármacos o algunas fibrosis familiares, entre otras, son casos donde resulta crucial establecer un diagnóstico certero debido a sus diferentes pronósticos respecto a FPI, y a la posibilidad de iniciar tratamientos específicos.

Las muestras de criobiopsia son más grandes que las obtenidas por fórceps y con menos defectos por aplastamiento. Como tal, esta técnica se ha centrado en el diagnóstico de EPID. La experiencia inicial ha sido favorable, y la mayoría de los informes sugieren que este procedimiento establece diagnósticos específicos de EPID en el 70-80\%, con mejor perfil de seguridad que $\mathrm{BPQ}^{37}$. 
Sin embargo, la literatura de criobiopsia existente está significativamente limitada por la falta de estandarización del procedimiento, puntos finales de diagnóstico variables, clasificación no uniforme sobre complicaciones y una metodología de estudio generalmente débil. No obstante, con la evidencia disponible actualmente, las criobiopsias parecen estar idealmente posicionadas para reemplazar las BPQ como la primera modalidad de biopsia en el algoritmo de diagnóstico de EPID ${ }^{37}$.

En pacientes con FPI avanzada se debe bloquear el bronquio principal del sitio donde se va a realizar la biopsia, durante un minuto antes del procedimiento, con el fin de evaluar si el paciente tolera ventilación unilateral en caso de que ocurra sangrado severo o neumotórax. Respecto a las exacerbaciones, estas han sido descritas principalmente con $\mathrm{BPQ}$, siendo poco frecuentes posterior a biopsia transbronquial o criobiopsia ${ }^{37}$.

\section{Crioterapia endobronquial en el manejo de obstrucción de la vía aérea}

La crioterapia tiene varias indicaciones con propósitos terapéuticos e incluyen toma de muestras de tumores endobronquiales, crio-desbridamiento en casos de tuberculosis endobronquial, manejo de estenosis traqueales y fibrosis endobronquial, remoción de cuerpos extraños y coágulos, criocirugía de cáncer de pulmón y crio-recanalización inmediata con resección completa de tumores endobronquiales $s^{31,38}$.

La crio-recanalización similar a la técnica de la crio-biopsia implica adhesión y extracción en bloque del tumor endobronquial a la criosonda. Pudiendo obtenerse efectos inmediatos, pero en ocasiones requiere de remoción de detritos o limpieza post-procedimiento.

La tasa de respuesta completa en estenosis bronquial tumoral es de $61 \%$ y $22 \%$ de respuesta parcial, con una tasa de respuesta general de $83 \%$, que en otros estudios ha sido descrita hasta 91\% cuando se realizan varias sesiones. La tasa de recaída a 6 meses puede ser hasta $24 \%$ y a 45 meses de $28 \%{ }^{22,39,40}$.

Después del procedimiento síntomas como disnea, tos y estridor muestran notoria mejoría, también en los estudios de función pulmonar (tanto CVF como $\mathrm{VEF}_{1}$ ).

\section{Criobiopsia en lesiones pulmonares periféricas}

La CBPTB puede ser útil en el estudio de lesiones pulmonares periféricas $<$ de $4 \mathrm{~cm}$, como lo describe Schuhmann y cols donde con ayuda de EBUS radial para posicionar la sonda, realizaron el procedimiento en 39 pacientes con un rendimiento diagnóstico de $74,2 \%{ }^{16,41}$.

\section{Criobiopsia en trasplante de pulmón}

Estudios de vigilancia y seguimiento de pacientes con trasplante de pulmón en los cuales hay indicación de broncoscopia post-trasplante incluyeron la realización de Biopsias transbronquiales y CBPTB. Se tomaron 2 a 3 muestras por criobiopsia, sin complicaciones mayores y obteniendo muestras con promedio de $10 \mathrm{~mm}$ de diámetro y $50 \mathrm{~mm}^{2}$ de área comparadas con $2 \mathrm{~mm}$ y $12,5 \mathrm{~mm}^{2}$ con biopsias trnasbronquiales; estas tenían un porcentaje mayor de tejido alveolar (65\% vs $35 \%$ ), logrando excluir o confirmar el diagnóstico de rechazo y estableciendo además otro tipo de diagnósticos.

Ninguno de los grupos presentó sangrado significativo (sin haber diferencias significativas) y tampoco se requirió el uso de balón de bloqueo bronquial. No hubo incidencia de neumotórax inmediato post biopsia en estos pacientes ${ }^{41,42}$.

\section{Criobiopsia en inmunocomprometidos con infiltrados pulmonares}

En este estudio piloto para evaluar la seguridad y eficacia de la CBPTB en inmunocomprometidos con infiltrados pulmonares la mayoría de ellos con inmunocompromiso de causa hematológica, el área promedio de la muestras fue $9 \mathrm{~mm}^{2}$ y en $70 \%$ de las muestras se logró presencia de tejido alveolar y no hubo complicaciones graves. El diagnostico obtenido por criobiopsia logro modificar el manejo en $80 \%$ de los $\operatorname{casos}^{43}$.

\section{Conclusiones}

La crio-tecnología, ha permitido implementar diversas técnicas que permiten el abordaje de diferentes patologías bronco-pulmonares, 
tanto diagnósticas como terapéuticas. En 50\% de las EPID no pueden ser clasificados con los datos clínico-tomográficos, requiriendo muestras del parénquima pulmonar para estudios de histopatología e inmunohistoquímica que nos permitan establecer el diagnóstico, tratamiento y pronóstico. La biopsia transbronquial no tiene buen rendimiento diagnóstico, razón por lo cual el estándar de oro histórico es la $\mathrm{BPQ}$, procedimiento que está asociado a mayor morbimortalidad, estancia hospitalaria y costos de atención. Con los datos disponibles en la actualidad, el rendimiento diagnóstico de la criobiopsia equiparable a la BPQ por VATS, asociado a la alta sensibilidad y especificidad con baja tasa de complicaciones y genera un ahorro económico considerable, por lo cual debe ser considerado como un paso previo a la BPQ en el diagnóstico y manejo de las EPID.

Si bien los estudios de CBPTB se han centrado en EPID, se debe avanzar en estudios de compromiso pulmonar en inmunosuprimidos, biopsia de lesiones periféricas únicas y analizar la realización de pruebas de biología molecular en muestras de criobiopsia y explorar el uso para microbiología. Quedando pendiente para la comunidad médica la realización de estudios prospectivos y aleatorizados.

\section{Referencias}

1. Bolliger CT, Mathur PN, Beamis JF, Becker HD, Cavaliere S, Colt H, et al. ERS/ATS statement on interventional pulmonology. European Respiratory Society/ American Thoracic Society. Eur Respir J 2002; 19 (2): 356-73.

2. Ong PG, Debiane LG, Casal RF. Recent advances in diagnostic bronchoscopy. J Thorac Dis 2016; 8 (12): 3808-17.

3. Poletti V, Casoni GL, Gurioli C, Ryu JH, Tomassetti S. Lung cryobiopsies: a paradigm shift in diagnostic bronchoscopy? Respirology 2014; 19 (5): 645-54.

4. Travis WD, Costabel U, Hansell DM, King TE, Jr., Lynch DA, Nicholson AG, et al. An official American Thoracic Society/European Respiratory Society statement: Update of the international multidisciplinary classification of the idiopathic interstitial pneumonias. Am J Respir Crit Care Med 2013; 188 (6): 733-48.

5. guideline TNIfHaCEIpfiadamC. nice.org.uk/guidance/ cg163 https://www.brit-thoracic.org.uk/.../guidelines/ bts-guideline 2013 .
6. Xaubet A, Ancochea J, Bollo E, Fernández-Fabrellas E, Franquet T, Molina-Molina M, et al. Guidelines for the diagnosis and treatment of idiopathic pulmonary fibrosis. Sociedad Espanola de Neumologia y Cirugia Toracica (SEPAR) Research Group on Diffuse Pulmonary Diseases. Arch Bronconeumol 2013; 49 (8): 343-53.

7. Sharp C, McCabe M, Adamali H, Medford AR. Use of transbronchial cryobiopsy in the diagnosis of interstitial lung disease-a systematic review and cost analysis. QJM 2017; 110 (4): 207-14.

8. Pajares V, Puzo C, Castillo D, Lerma E, Montero MA, Ramos-Barbon D, et al. Diagnostic yield of transbronchial cryobiopsy in interstitial lung disease: a randomized trial. Respirology 2014; 19 (6): 900-6.

9. Kondoh Y, Taniguchi H, Kitaichi M, Yokoi T, Johkoh T, Oishi T, et al. Acute exacerbation of interstitial pneumonia following surgical lung biopsy. Respir Med 2006; 100 (10): 1753-9.

10. Utz JP, Ryu JH, Douglas WW, Hartman TE, Tazelaar HD, Myers JL, et al. High short-term mortality following lung biopsy for usual interstitial pneumonia. Eur Respir J 2001; 17 (2): 175-9.

11. Lettieri CJ, Veerappan GR, Helman DL, Mulligan CR, Shorr AF. Outcomes and safety of surgical lung biopsy for interstitial lung disease. Chest 2005; 127 (5): 1600-5.

12. Hagmeyer L, Theegarten D, Wohlschlager J, Treml M, Matthes S, Priegnitz C, et al. The role of transbronchial cryobiopsy and surgical lung biopsy in the diagnostic algorithm of interstitial lung disease. Clin Respir J 2016; 10 (5): 589-95.

13. Maldonado F, Moua T, Skalski J. Parenchymal cryobiopsies for interstitial lung diseases: a step forward in disease management. Respirology 2014; 19 (6): 773-4.

14. Ganganah O, Guo SL, Chiniah M, Li YS. Efficacy and safety of cryobiopsy versus forceps biopsy for interstitial lung diseases and lung tumours: A systematic review and meta-analysis. Respirology 2016; 21 (5): 834-41.

15. Johannson KA, Marcoux VS, Ronksley PE, Ryerson CJ. Diagnostic Yield and Complications of Transbronchial Lung Cryobiopsy for Interstitial Lung Disease. A Systematic Review and Metaanalysis. Ann Am Thorac Soc 2016; 13 (10): 1828-38.

16. Casoni GL, Tomassetti S, Cavazza A, Colby TV, Dubini A, Ryu JH, et al. Transbronchial lung cryobiopsy in the diagnosis of fibrotic interstitial lung diseases. PLoS One 2014; 9 (2): e86716.

17. Pajares Ruiz V, Torrego Fernández A, Puzo Ardanuy C, Gil de Bernabe A. Use of an occlusion balloon in transbronchial lung cryobiopsy. Arch Bronconeumol 2014; 50 (7): 309-10.

18. Ussavarungsi K, Kern RM, Roden AC, Ryu JH, Edell 
ES. Transbronchial Cryobiopsy in Diffuse Parenchymal Lung Disease: Retrospective Analysis of 74 Cases. Chest 2017; 151 (2): 400-8.

19. Tomassetti S, Piciucchi S, Tantalocco P, Dubini A, Poletti V. The multidisciplinary approach in the diagnosis of idiopathic pulmonary fibrosis: a patient case-based review. Eur Respir Rev 2015; 24 (135): 69-77.

20. Pajares VTA. Criobiopsia pulmonar transbronquial: procedimiento e indicaciones. Medicina Respiratoria 2016; 9 (6): 51-60.

21. Tomic R, Cortés-Puentes GA, Murugan P, Joo Kim H, Amin K, Dincer HE. Acute Exacerbation of Interstitial Lung Disease After Cryobiopsy. J Bronchology Interv Pulmonol 2017; 24 (4): 319-22.

22. Schuhmann M, Bostanci K, Bugalho A, Warth A, Schnabel PA, Herth FJ, et al. Endobronchial ultrasound-guided cryobiopsies in peripheral pulmonary lesions: a feasibility study. Eur Respir J 2014; 43 (1): 233-9.

23. Molina M BA. Manual 24 de Procedimientos en biopsia pulmonar para el diagnóstico de PEID. SEPAR, editor 2013.

24. Babiak A, Hetzel J, Krishna G, Fritz P, Moeller P, Balli $\mathrm{T}$, et al. Transbronchial cryobiopsy: a new tool for lung biopsies. Respiration 2009; 78 (2): 203-8.

25. Griff S, Schonfeld N, Ammenwerth W, Blum TG, Grah C, Bauer TT, et al. Diagnostic yield of transbronchial cryobiopsy in non-neoplastic lung disease: a retrospective case series. BMC Pulm Med 2014; 14: 171.

26. Fruchter O, Fridel L, El Raouf BA, Abdel-Rahman N, Rosengarten D, Kramer MR. Histological diagnosis of interstitial lung diseases by cryo-transbronchial biopsy. Respirology 2014; 19 (5): 683-8.

27. Hernández-González F, Lucena CM, Ramírez J, Sánchez M, Jiménez MJ, Xaubet A, et al. Cryobiopsy in the diagnosis of diffuse interstitial lung disease: yield and cost-effectiveness analysis. Arch Bronconeumol 2015; 51 (6): 261-7.

28. Kropski JA, Pritchett JM, Mason WR, Sivarajan L, Gleaves LA, Johnson JE, et al. Bronchoscopic cryobiopsy for the diagnosis of diffuse parenchymal lung disease. PLoS One 2013; 8 (11): e78674.

29. Berbescu EA, Katzenstein AL, Snow JL, Zisman DA. Transbronchial biopsy in usual interstitial pneumonia. Chest 2006; 129 (5): 1126-31.

30. Iftikhar IH, Alghothani L, Sardi A, Berkowitz D, Musani AI. Transbronchial Lung Cryobiopsy and Video-assisted Thoracoscopic Lung Biopsy in the Diagnosis of Diffuse Parenchymal Lung Disease. A Meta-analysis of Diagnostic Test Accuracy. Ann Am Thorac Soc 2017; 14 (7):
1197-211.

31. Fu EQ, Nan YD, Jin FG, Ma AQ. Therapeutic effects of sequential therapy by electric coagulation, cryotherapy and balloon dilation with an electronic video bronchoscope. Exp Ther Med 2013; 5 (6): 1649-56.

32. Shim HS, Park MS, Park IK. Histopathologic findings of transbronchial biopsy in usual interstitial pneumonia. Pathol Int 2010; 60 (5): 373-7.

33. Tomassetti S, Cavazza A, Colby TV, Ryu JH, Nanni O, Scarpi E, et al. Transbronchial biopsy is useful in predicting UIP pattern. Respir Res 2012;13:96.

34. Kaarteenaho R. The current position of surgical lung biopsy in the diagnosis of idiopathic pulmonary fibrosis. Respir Res 2013; 14: 43.

35. Maguire MF, Ravenscroft A, Beggs D, Duffy JP. A questionnaire study investigating the prevalence of the neuropathic component of chronic pain after thoracic surgery. Eur J Cardiothorac Surg 2006; 29 (5): 800-5.

36. Lee SH, Choi WJ, Sung SW, Kim YK, Kim CH, Zo JI, et al. Endoscopic cryotherapy of lung and bronchial tumors: a systematic review. Korean J Intern Med 2011; 26 (2): 137-44.

37. Ravaglia C, Bonifazi M, Wells AU, Tomassetti S, Gurioli C, Piciucchi S, et al. Safety and Diagnostic Yield of Transbronchial Lung Cryobiopsy in Diffuse Parenchymal Lung Diseases: A Comparative Study versus Video-Assisted Thoracoscopic Lung Biopsy and a Systematic Review of the Literature. Respiration 2016; 91 (3): 215-27.

38. Hetzel M, Hetzel J, Schumann C, Marx N, Babiak A. Cryorecanalization: a new approach for the immediate management of acute airway obstruction. J Thorac Cardiovasc Surg 2004; 127 (5): 1427-31.

39. Homasson JP, Renault P, Angebault M, Bonniot JP, Bell NJ. Bronchoscopic cryotherapy for airway strictures caused by tumors. Chest 1986; 90 (2): 159-64.

40. Deygas N, Froudarakis M, Ozenne G, Vergnon JM. Cryotherapy in early superficial bronchogenic carcinoma. Chest 2001; 120 (1): 26-31.

41. Fruchter O, Fridel L, Rosengarten D, Raviv Y, Rosanov V, Kramer MR. Transbronchial cryo-biopsy in lung transplantation patients: first report. Respirology 2013; 18 (4): 669-73.

42. Yarmus L, Akulian J, Gilbert C, Illei P, Shah P, Merlo C, et al. Cryoprobe transbronchial lung biopsy in patients after lung transplantation: a pilot safety study. Chest 2013; 143 (3): 621-6.

43. Chen AC, Feller-Kopman D. Cryobiopsy: A Work in Progress. Ann Am Thorac Soc 2017; 14 (6): 827-8. 